\title{
Female Genital Tuberculosis Among Infertile Women and Its Contributions to Primary and Secondary Infertility A systematic review and meta-analysis
}

"Musa A.E. Ahmed, ${ }^{1,3}$ Abdullah A.A. Mohammed ${ }^{1,4}$ Abiodun O. Ilesanmi, ${ }^{2}$ Christopher O. Aimakhu, ${ }^{2}$ Amel O. Bakhiet, ${ }^{5,6}$ Suad B.M. Hamad ${ }^{7}$

\begin{abstract}
Female genital tuberculosis (FGTB) is a widespread infectious disease among young women. This meta-analysis aimed to investigate the prevalence of FGTB among infertile women and its contribution to primary and secondary infertility. PubMed, MEDLINE ${ }^{\circledast}$, WorldCat, The Lens, direct Google search, Google Scholar and ResearchGate were searched from 1971 to July 17, 2021 using the following terms: "prevalence", "epidemiology", "urogenital tuberculosis", "FGTB", "infertile women", "infertility complaints" and "FGTB testing methods". Data were extracted and a meta-analysis was performed. A total of 42 studies were selected with a total of 30,918 infertile women. Of these, the pooled prevalence of FGTB was $20 \%$ (95\% confidence interval: $15-25 \%, \mathrm{I}^{2}=99.94 \%$ ) and the prevalence of overall infertility, primary infertility and secondary infertility among FGTB population were $88 \%$, $66 \%$ and $34 \%$, respectively. The proportion of FGTB is remarkable among infertile women globally. The biggest burden of the disease is present in low-income countries followed by lower-to-middle- and upper-to-middle-income countries.
\end{abstract}

Keywords: Female Genital Tuberculosis; Female Infertility; Worldwide; Prevalence of FGTB; Mycobacterium Tuberculosis; Infertility.

$\mathrm{T}$

UBERCULOSIS (TB) IS AN INFECTIOUS DISEASE caused by Mycobacterium tuberculosis which was recently listed among the top 10 diseases causing death around the world. According to the World Health Organization (WHO), 10.0 million people were infected with TB and there were 1.2 million TB deaths in 2019. ${ }^{1}$ Two-thirds of this global burden was present in eight countries - India, Indonesia, China, the Philippines, Pakistan, Nigeria, Bangladesh and South Africa. ${ }^{1}$ Female genital tuberculosis (FGTB) is commonly secondary to pulmonary $\mathrm{TB}$ (PTB) or extrapulmonary $\mathrm{TB}$ (EPTB), with an incidence rate ranging between 9-20\% and 5-13\% among overall EPTB and PTB cases worldwide according to many multitask studies, respectively. ${ }^{2-6}$ Typically, FGTB is known as a disease of young women (20-40 years old); 5,7 it is usually diagnosed during infertility evaluations. ${ }^{2,8}$ A previous study indicated that the infertility rates in women is higher compared to men. ${ }^{9}$ Moreover, $76 \%$ of infertile women had a history of $\mathrm{TB} ;{ }^{10}$ infertility is the most frequent complaint of FGTB cases which occurs due to irreversible damage to the fallopian tube., ${ }^{4,11}$ In addition to infertility, other clinical presentations of FGTB include pelvic pain or menstrual irregularities, and it remains a major health problem in low-income countries. ${ }^{8,12}$ Organs commonly affected by FGTB are the fallopian tube (90\%), ovaries (10-30\%), endometrium (50\%), cervix and vagina. ${ }^{3,13}$ Infertile FGTB patients have been reported to have a longer duration of infertility compared to infertility from other sources. ${ }^{14}$

This meta-analysis was conducted to investigate the prevalence of FGTB among infertile women of reproductive age and to evaluate the incidence of primary and secondary infertility among FGTB patients around the globe.

\section{Methods}

\section{ELIGIBILITY CRITERIA}

Studies were eligible if they characterised the epidemiology of FGTB among women within reproductive age, if the study population was infertile women or at least indicated a proportion of infertility complaints with enough explanation of epidemiology of FGTB, were published in English, were published between 1971 to 17 July, 2021 and used the diagnostic methods of FGTB based on the particular infertility centres testing protocol. Articles were excluded if they were characterised as only PTB or EPTB regardless of FGTB and if the reported prevalence of FGTB was not that of infertile women. 


\section{INFORMATION SOURCES}

This study was carried out in accordance with the guidelines of Preferred Reporting Items for Systematic Reviews and Meta-Analyses (PRISMA). Several electronic databases such as MEDLINE ${ }^{\circledR}$ (National Library of Medicine, Bethesda, Maryland, USA), WorldCat (OCLC, Inc., Dublin, Ohio, USA), The Lens (Cambia, Canberra, Australia) and PubMed (National Library of Medicine) were used to retrieve published articles. In addition, other search engines were intensively combed through including direct Google search (Google LLC, Mountain View, California, USA), Google Scholar (Google LLC) and ResearchGate, in order to retrieve studies that were not indexed in PubMed. All mentioned databases were searched from their commencement for the period between 1971 to July 17, 2021, for human studies published in English.

\section{SEARCH STRATEGY}

The Boolean search terms (AND, OR) were used to develop the research strategy to retrieve studies from PubMed and WorldCat. The final search strategy included the use of title/abstract related to ([Female genital tuberculosis] OR [urogenital tuberculosis]) AND ([prevalence] OR [epidemiology]) AND ([infertile women] OR [infertility]). Manual searches were applied in a direct Google search, Google Scholar and ResearchGate for the same purpose.

\section{STUDY SELECTION PROCESS}

All retrieved articles were first screened by title and abstract followed by a full-text screening. Then, eligible articles were exported to the Mendeley citation manager software (Elsevier, Amsterdam, the Netherlands), Version 1.19.8, to check for duplicates; all duplicated articles were removed. Two authors (AM and MA) screened and evaluated the remaining studies independently by a careful reading of the title and abstract followed by a full-text article screening if the particular records mentioned the outcomes of the review 'Prevalence of Female genital tuberculosis among infertile women' in their titles and abstract. However, the screened full-text articles were considered for further evaluation based on the objectives, methods, participants and key findings. The two authors (MA and AM) independently evaluated the quality of the studies using the PRISMA checklist. ${ }^{15}$ Any inconsistency for the included articles was resolved through discussion and by consulting an expert. The overall study selection process is presented using the PRISMA statement flow diagram [Figure 1].

\section{DATA COLLECTION PROCESS}

The relevant data from selected articles were extracted by three investigators independently (AM, MA and $\mathrm{SH}$ ) using a data extraction template by Microsoft Office Word, Version 2016 (Microsoft Corp., Redmond, Washington, USA). The extracted information included author name, year of publication, reference, study country, study design/setting, sample size, FGTB proportion among infertile women, the prevalence of overall infertility, primary infertility and secondary infertility among FGTB cases [Table 1]. The data extraction accuracy was verified by comparing the data extraction results by a second group of investigators $(\mathrm{AB}, \mathrm{AI}$ and $\mathrm{CA})$, who independently extracted the data in a randomly-selected subset of papers (30\% of the total). The extracted quantitative data were summarised in a Microsoft Excel sheet (Microsoft Corp.). Based on the aim of this study, which is 'to investigate the pooled prevalence of FGTB among infertile women globally', the collected data was divided into four subgroups according to the World Bank economical classification. The prevalence of FGTB among infertile women and prevalence of pooled infertility (primary and secondary) among FGTB cases were calculated using STATA, Version 16 (StataCorp LP, College Station, Texas, USA).

\section{DATA ITEMS}

The main outcome of this study was the prevalence of FGTB among infertile women within reproductive age worldwide which is measured by the direct reports from individual studies. Out of these, 26 studies from India, three studies from Nigeria and two articles each from Ethiopia, South Africa and Pakistan were retrieved. Also, only one article each was retrieved from Egypt, Iraq, Iran, USA, Saudi Arabia, Sudan and Yemen. To quantify the outcome, the investigators considered studies that reported the prevalence of FGTB among infertile women and the types of TB regarding FGTB among gynaecology-admitted/infertile women in their statistics. The result was interpreted by the proportions of the infertile population having any type of FGTB from the total population studied.

\section{STUDY RISK OF BIAS ASSESSMENT}

Inclusion criteria were appraised for all retrieved articles by using the title and abstract; then, full-text articles were screened to check the quality of each study before final selection. The quality assessment criteria for the studies included in the current metaanalysis and systematic review is as follows: (1) the 


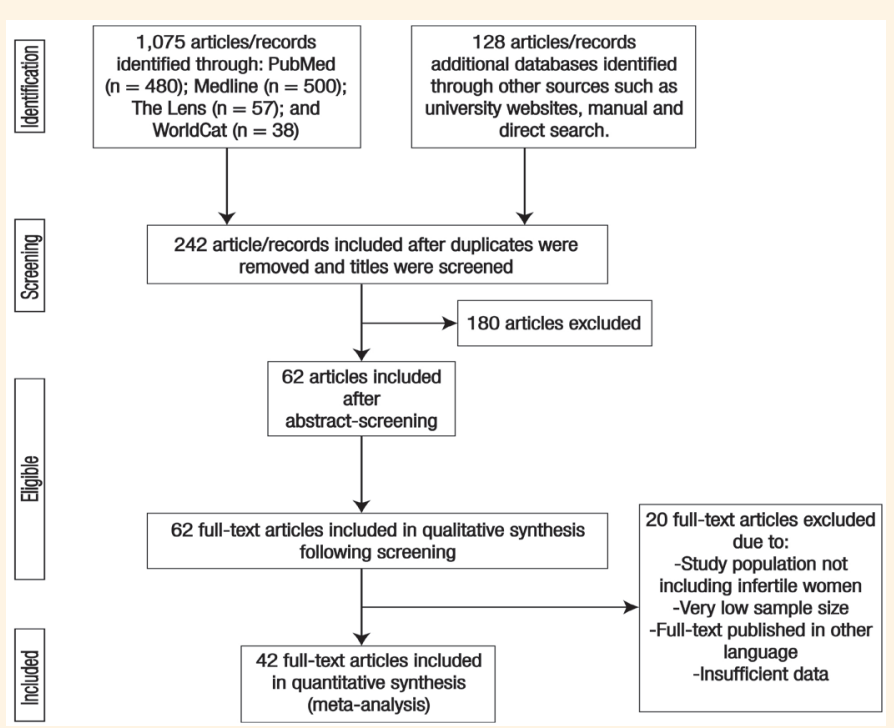

Figure 1: Flowchart showing the selection process for articles included in the current review.

diagnosis of the infertility cases were performed at an infertility centre with consideration that infertility is defined as one year without conception after unprotected intercourse; (2) the infertility was not due to the male factor; (3) the diagnosis included an infertile population who tested for FGTB willingly; (4) the diagnosis of FGTB was conducted after excluding the patients with confirmed FGTB; and (5) the sample size was representative of the population. A comprehensive search including electronic databases, grey literature and unpublished studies was done in order to manage and minimise the risk of bias. Moreover, two groups of investigators (AM, MA and $\mathrm{SH}$ ) and $(\mathrm{AB}, \mathrm{AI}$ and $\mathrm{CA})$ used the Joanna Briggs Institute Quality Assessment Tool as a critical appraisal tool. ${ }^{16}$ The differences in the inclusion of the studies were resolved by consensus. The included studies were evaluated against each indicator of the tool and categorised as high-, moderate- and lowquality. Studies with a score $\geq 60 \%$ were included. The publication bias for the included studies was checked by both the visual inspection of the funnel plot and the statistical symmetry of the funnel plot using Egger's regression test.

\section{SUMMARY MEASURES}

The proportion of FGTB among infertile women and proportion of the type of infertility among FGTB patients were used to synthesise and present the results for the analysis.

\section{SYNTHESIS METHODS}

The collected data were synthesised and analysed in STATA (StataCorp LP). The recommendations of the $\mathrm{I}^{2}$ statistic described by Higgins et al. (an I $\mathrm{I}^{2}$ of $75 / 100 \%$ and above suggests considerable heterogeneity) were used to perform this meta-analysis. ${ }^{17}$ The effect size, with a 95\% confidence interval (CI) and standard error (SE), was used to calculate the results of this study. The effect size of this study was the prevalence of FGTB and the prevalence of the type of infertility subgroups; these were calculated using the binomial distribution. The SE was calculated using the sample size (n) and the proportion of FGTB (p) using the following equation:

$$
\sqrt{ } P(1-P) \div \eta[\text { Equation 1] }
$$

Any potential publication bias was checked using a funnel plot and Egger's regression test. Subgroup analysis was applied to check the potential source of heterogeneity and possible source of bias. Any study with missing data and/or a high risk of bias was excluded. $P$ values were interpreted as statistically significant at a value of less than 0.10 . The study results were reported according to the PRISMA guidelines and the findings were presented using a narrative synthesis followed by a meta-analysis chart.

\section{Results}

\section{STUDY SELECTION}

A total of 1,203 articles/records were identified. Of these, 961 articles/records were removed due to duplication and title screening resulting in 242 articles/records for further inspection. Subsequently, another 180 articles were excluded after a very careful screening of abstracts. Therefore, a total of 62 articles were eligible for full-text screening; 20 articles of these were excluded due to inconsistency with the study inclusion criteria. Finally, 42 articles fulfilled 
Table 1: Characteristics of studies included in the current meta-analysis ${ }^{4-7,10,12,19,20,23-28,38-64}$

\begin{tabular}{|c|c|c|c|c|c|c|c|c|c|}
\hline \multirow{3}{*}{$\begin{array}{l}\text { Author } \\
\text { and year of } \\
\text { publication }\end{array}$} & \multirow{3}{*}{$\begin{array}{l}\text { Study } \\
\text { design/ } \\
\text { setting }\end{array}$} & \multirow{3}{*}{$\begin{array}{l}\text { World Bank } \\
\text { country } \\
\text { income } \\
\text { classification }\end{array}$} & \multirow[t]{3}{*}{ Country } & \multirow{3}{*}{$\begin{array}{l}\text { Infertile } \\
\text { population }\end{array}$} & \multirow{3}{*}{$\begin{array}{l}\text { FGTB testing } \\
\text { method }\end{array}$} & \multicolumn{4}{|c|}{ n (\%) } \\
\hline & & & & & & \multirow[t]{2}{*}{$\begin{array}{l}\text { Proportion } \\
\text { of FGTB }\end{array}$} & \multicolumn{3}{|c|}{$\begin{array}{l}\text { Proportion of infertility among } \\
\text { FGTB patient }\end{array}$} \\
\hline & & & & & & & $\begin{array}{l}\text { Overall } \\
\text { infertility }\end{array}$ & PI & SI \\
\hline $\begin{array}{l}\text { Chattopadhyay } \\
\text { et al. } .^{38}(1986)\end{array}$ & $\mathrm{CS} / \mathrm{HA}$ & High-income & $\begin{array}{l}\text { Saudi } \\
\text { Arabia }\end{array}$ & 945 & NA & $40(4.2)$ & NA & NA & NA \\
\hline $\begin{array}{l}\text { Tal et al. }{ }^{39} \\
(2020)\end{array}$ & $\mathrm{PC} / \mathrm{HC}$ & High-income & USA & 323 & $\begin{array}{l}\text { QuantiFERON- } \\
\text { TB }\end{array}$ & $25(7.7)$ & $\mathrm{NA}$ & NA & $\mathrm{NA}$ \\
\hline $\begin{array}{l}\text { Abdissa et al. }{ }^{4} \\
(2018)\end{array}$ & $\mathrm{CS} / \mathrm{HA}$ & Low-income & Ethiopia & 152 & PCR, CP, HE & $8(5.3)$ & $5(62.5)$ & $4(50)$ & $1(12.5)$ \\
\hline $\begin{array}{l}\text { Abebe et al. }{ }^{13} \\
(2004)\end{array}$ & $\mathrm{CS} / \mathrm{HA}$ & Low-income & Ethiopia & 25 & $\begin{array}{l}\text { AFB, CP, HE, } \\
\quad \text { PCR }\end{array}$ & $16(64)$ & NA & NA & $\mathrm{NA}$ \\
\hline $\begin{array}{l}\text { Ali and } \\
\text { Abdallah }^{40} \\
(2012)\end{array}$ & $\mathrm{CS} / \mathrm{HA}$ & Low-income & Sudan & 2,778 & $\mathrm{HE}$ & $25(0.9)$ & $\mathrm{NA}$ & NA & NA \\
\hline $\begin{array}{l}\text { Abdelrub and } \\
\text { Al Harazi }{ }^{41} \\
(2015)\end{array}$ & $\mathrm{P} / \mathrm{O} / \mathrm{HA}$ & Low-income & Yemen & 151 & $\begin{array}{c}\text { AFB, PCR, CP, } \\
\text { HE }\end{array}$ & $47(31.1)$ & NA & NA & NA \\
\hline $\begin{array}{l}\text { Nezar et al. } .^{42} \\
(2009)\end{array}$ & $\mathrm{P} / \mathrm{O} / \mathrm{HA}$ & $\begin{array}{l}\text { Lower-middle } \\
\text { income }\end{array}$ & Egypt & 420 & $\begin{array}{l}\text { Laparoscopy, } \\
\text { HE, PCR }\end{array}$ & $24(5.7)$ & $24(100)$ & NA & NA \\
\hline $\begin{array}{l}\text { Kumar et al. }{ }^{6} \\
\text { (2008) }\end{array}$ & $\mathrm{CS} / \mathrm{HA}$ & $\begin{array}{l}\text { Lower-middle } \\
\text { income }\end{array}$ & India & 285 & PCR & $111(39)$ & $111(100)$ & NA & NA \\
\hline $\begin{array}{l}\text { Mohakul et al. } \\
\text { (2015) }\end{array}$ & $\mathrm{P} / \mathrm{HC}$ & $\begin{array}{l}\text { Lower-middle } \\
\text { income }\end{array}$ & India & 105 & $\begin{array}{c}\text { PCR, } \\
\text { hysteroscopy }\end{array}$ & $41(39)$ & $41(100)$ & $24(58)$ & $17(42)$ \\
\hline Jindal ${ }^{10}(2006)$ & $\mathrm{R} / \mathrm{HC}$ & $\begin{array}{l}\text { Lower-middle } \\
\text { income }\end{array}$ & India & 2,083 & $\begin{array}{l}\text { LAP, AFB, HE, } \\
\text { MT, ELISA }\end{array}$ & $10(7.2)$ & $146(97.3)$ & $\begin{array}{l}105 \\
(70)\end{array}$ & $41(27.3)$ \\
\hline $\begin{array}{l}\text { Singh et al. }{ }^{43} \\
(2008\end{array}$ & $\mathrm{R} / \mathrm{HC}$ & $\begin{array}{l}\text { Lower-middle } \\
\text { income }\end{array}$ & India & 140 & $\begin{array}{c}\text { MH, } \\
\text { laparoscopy, } \\
\text { hysteroscopy }\end{array}$ & $34(48.5)$ & NA & NA & NA \\
\hline $\begin{array}{l}\text { Sankar et al. }{ }^{44} \\
(2013)\end{array}$ & $\mathrm{R} / \mathrm{HA}$ & $\begin{array}{l}\text { Lower-middle } \\
\text { income }\end{array}$ & India & 620 & $\begin{array}{l}\text { AFB, PCR, CP, } \\
\text { HE }\end{array}$ & $158(25.5)$ & 151 (95.5) & $\begin{array}{c}119 \\
(78.8)\end{array}$ & $32(21.2)$ \\
\hline $\begin{array}{l}\text { Mahajan et al. }{ }^{45} \\
(2016)\end{array}$ & $\mathrm{CS} / \mathrm{HA}$ & $\begin{array}{l}\text { Lower-middle } \\
\text { income }\end{array}$ & India & 180 & PCR, CP & $74(41)$ & NA & NA & NA \\
\hline $\begin{array}{l}\text { Sethi et al. }{ }^{46} \\
(2016)\end{array}$ & $\mathrm{CS} / \mathrm{HA}$ & $\begin{array}{l}\text { Lower-middle } \\
\text { income }\end{array}$ & India & 300 & $\begin{array}{c}\text { AFB, PCR, CP, } \\
\text { HE }\end{array}$ & $68(22.7)$ & $\mathrm{NA}$ & NA & $\mathrm{NA}$ \\
\hline $\begin{array}{l}\text { Chatterjee et } \\
\text { al. } .^{5}(2018)\end{array}$ & $\mathrm{CS} / \mathrm{HA}$ & $\begin{array}{l}\text { Lower-middle } \\
\text { income }\end{array}$ & India & 120 & PCR & $2(1.7)$ & NA & NA & NA \\
\hline $\begin{array}{l}\text { Chowdhury et } \\
\text { al. }^{47}(2010)\end{array}$ & $\mathrm{CO} / \mathrm{HA}$ & $\begin{array}{l}\text { Lower-middle } \\
\text { income }\end{array}$ & India & 517 & PCR & $230(44.5)$ & $114(49.7)$ & NA & NA \\
\hline $\begin{array}{l}\text { Saraswat et al. }{ }^{48} \\
(2010)\end{array}$ & $\mathrm{CS} / \mathrm{HA}$ & $\begin{array}{l}\text { Lower-middle } \\
\text { income }\end{array}$ & India & 125 & PCR, CP & $26(20.8)$ & NA & NA & NA \\
\hline $\begin{array}{l}\text { Malhotra et } \\
\text { al. }{ }^{99}(2012)\end{array}$ & $\mathrm{O} / \mathrm{HA}$ & $\begin{array}{l}\text { Lower-middle } \\
\text { income }\end{array}$ & India & 555 & AFB, PCR, CP & $140(25.22)$ & $\mathrm{NA}$ & $\mathrm{NA}$ & NA \\
\hline $\begin{array}{l}\text { GajBhIye et } \\
\text { al. } .^{50}(2019)\end{array}$ & $\mathrm{CS} / \mathrm{O} / \mathrm{HA}$ & $\begin{array}{l}\text { Lower-middle } \\
\text { income }\end{array}$ & India & 50 & PCR & $6(12)$ & $5(83.3)$ & $4(80)$ & $1(20)$ \\
\hline $\begin{array}{l}\text { Bhanothu et } \\
\text { al. } .^{1}(2014)\end{array}$ & $\mathrm{P} / \mathrm{CC} / \mathrm{HA}$ & $\begin{array}{l}\text { Lower-middle } \\
\text { income }\end{array}$ & India & 302 & PCR & $86(28.47)$ & $\mathrm{NA}$ & $\mathrm{NA}$ & NA \\
\hline $\begin{array}{l}\text { Gurjar et al. } .^{20} \\
\text { (2018) }\end{array}$ & $\mathrm{O} / \mathrm{HA}$ & $\begin{array}{l}\text { Lower-middle } \\
\text { income }\end{array}$ & India & 100 & PCR & $52(52)$ & $\mathrm{NA}$ & $\mathrm{NA}$ & NA \\
\hline
\end{tabular}

FGTB = female genital tuberculosis; $P I=$ primary infertility; $S I=$ secondary infertility; $C S=$ cross-sectional study; $H A=$ hospital admitted patients; NA = Not available; $P C=$ prospective cohort study; $H C=$ infertility centre admitted patient; $P C R=$ polymerase chain reaction test; $C P=$ culture proven; HE = histopathological examination; $A F B=$ acid-fast bacilli test $P=$ prospective study; $O=$ observational study; $R=$ retrospective study; $L A P=$ laparotomy; $M T$ = Mantoux test; ELISA = enzyme-linked immunosorbent assay; $M H=$ menstrual history; $C C=$ case control study; $M T D=$ Mycobacterium Tuberculosis Direct test; $C B N A A T=$ cartridge based nucleic acid amplification test; $Z N=$ Ziehl-Neelsen (ZN) smear microscopy. 
Table 1 (cont'd.): Characteristics of studies included in the current meta-analysis ${ }^{4-7,10,12,19,20,23-28,38-64}$

\begin{tabular}{|c|c|c|c|c|c|c|c|c|c|}
\hline \multirow{3}{*}{$\begin{array}{l}\text { Author } \\
\text { and year of } \\
\text { publication }\end{array}$} & \multirow{3}{*}{$\begin{array}{l}\text { Study } \\
\text { design/ } \\
\text { setting }\end{array}$} & \multirow{3}{*}{$\begin{array}{l}\text { World Bank } \\
\text { country } \\
\text { income } \\
\text { classification }\end{array}$} & \multirow[t]{3}{*}{ Country } & \multirow{3}{*}{$\begin{array}{l}\text { Infertile } \\
\text { population }\end{array}$} & \multirow{3}{*}{$\begin{array}{l}\text { FGTB testing } \\
\text { method }\end{array}$} & \multicolumn{4}{|c|}{ n (\%) } \\
\hline & & & & & & \multirow[t]{2}{*}{$\begin{array}{c}\text { Proportion } \\
\text { of FGTB }\end{array}$} & \multicolumn{3}{|c|}{$\begin{array}{l}\text { Proportion of infertility among } \\
\text { FGTB patient }\end{array}$} \\
\hline & & & & & & & $\begin{array}{l}\text { Overall } \\
\text { infertility }\end{array}$ & PI & SI \\
\hline $\begin{array}{l}\text { Patil et al. } .^{52} \\
(2015)\end{array}$ & CS/HA & $\begin{array}{l}\text { Lower-middle } \\
\text { income }\end{array}$ & India & 123 & $\begin{array}{l}\text { Gen-Probe } \\
\text { MTD test }\end{array}$ & $1(0.8)$ & NA & NA & NA \\
\hline $\begin{array}{l}\text { Goel et al. }{ }^{53} \\
(2013)\end{array}$ & $\mathrm{R} / \mathrm{HA}$ & $\begin{array}{l}\text { Lower-middle } \\
\text { income }\end{array}$ & India & 546 & PCR & $20(3.7)$ & NA & NA & NA \\
\hline $\begin{array}{l}\text { Kamal et al. } .^{54} \\
(2020)\end{array}$ & $\mathrm{P} / \mathrm{HA}$ & $\begin{array}{l}\text { Lower-middle } \\
\text { income }\end{array}$ & India & 100 & PCR, HE & $27(27)$ & $\mathrm{NA}$ & $\begin{array}{c}16 \\
(59.4)\end{array}$ & $11(40.6)$ \\
\hline $\begin{array}{l}\text { Kanti V et al. }{ }^{55} \\
(2021)\end{array}$ & $\mathrm{P} / \mathrm{HC}$ & $\begin{array}{l}\text { Lower-middle } \\
\text { income }\end{array}$ & India & 59 & CBNAAT, HE & $2(3.4)$ & $\mathrm{NA}$ & $2(100)$ & 0 \\
\hline $\begin{array}{l}\text { Meenu et al. }{ }^{56} \\
(2020)\end{array}$ & $\mathrm{CS} / \mathrm{HA}$ & $\begin{array}{l}\text { Lower-middle } \\
\text { income }\end{array}$ & India & 139 & PCR & $58(41.7)$ & $\mathrm{NA}$ & NA & NA \\
\hline $\begin{array}{l}\text { Shende et al. }{ }^{57} \\
\text { (2017) }\end{array}$ & $\mathrm{P} / \mathrm{HA}$ & $\begin{array}{l}\text { Lower-middle } \\
\text { income }\end{array}$ & India & 120 & PCR & $32(27)$ & NA & NA & NA \\
\hline $\begin{array}{l}\text { Shrivastava \& } \\
\operatorname{Patel}^{7}(2014)\end{array}$ & $\mathrm{P} / \mathrm{HC}$ & $\begin{array}{l}\text { Lower-middle } \\
\text { income }\end{array}$ & India & 218 & $\begin{array}{l}\text { AFB, CP, HE, } \\
\text { PCR }\end{array}$ & $86(39.45)$ & NA & NA & NA \\
\hline $\begin{array}{l}\text { Ohri et al. }{ }^{58} \\
\text { (2016) }\end{array}$ & $\mathrm{P} / \mathrm{HA}$ & $\begin{array}{l}\text { Lower-middle } \\
\text { income }\end{array}$ & India & 50 & PCR & $9(18)$ & $\mathrm{NA}$ & $\begin{array}{c}8 \\
(88.9)\end{array}$ & $1(11.1)$ \\
\hline $\begin{array}{l}\text { Parikh et al. }{ }^{59} \\
(2014)\end{array}$ & $\mathrm{P} / \mathrm{HA}$ & $\begin{array}{l}\text { Lower-middle } \\
\text { income }\end{array}$ & India & 50 & PCR & $6(12)$ & $\mathrm{NA}$ & $3(50)$ & $3(50)$ \\
\hline $\begin{array}{l}\text { Gupta et al. }{ }^{60} \\
(2007)\end{array}$ & $\mathrm{R} / \mathrm{HA}$ & $\begin{array}{l}\text { Lower-middle } \\
\text { income }\end{array}$ & India & 150 & AFB, MT, PCR & $40(26.7)$ & NA & $30(75)$ & $10(25)$ \\
\hline $\begin{array}{l}\text { Rajaram et al. }{ }^{61} \\
(2016)\end{array}$ & $\mathrm{PC} / \mathrm{HA}$ & $\begin{array}{l}\text { Lower-middle } \\
\text { income }\end{array}$ & India & 50 & HE, PCR & $14(28)$ & $\mathrm{NA}$ & NA & NA \\
\hline $\begin{array}{l}\text { Ojo et al. }{ }^{19} \\
\text { (2008) }\end{array}$ & $\mathrm{R} / \mathrm{HA}$ & $\begin{array}{l}\text { Lower-middle } \\
\text { income }\end{array}$ & Nigeria & 661 & AFB, HE & $3(0.45)$ & NA & $\begin{array}{c}1 \\
(33.3)\end{array}$ & $2(66.7)$ \\
\hline $\begin{array}{l}\text { Ojo et al. } .^{23} \\
(1971)\end{array}$ & $\mathrm{CS} / \mathrm{HA}$ & $\begin{array}{l}\text { Lower-middle } \\
\text { income }\end{array}$ & Nigeria & 11,896 & $\mathrm{HE}$ & $82(0.7)$ & NA & NA & NA \\
\hline $\begin{array}{l}\text { Emembolu }{ }^{28} \\
\text { (1989) }\end{array}$ & $\mathrm{R} / \mathrm{HA}$ & $\begin{array}{l}\text { Lower-middle } \\
\text { income }\end{array}$ & Nigeria & 114 & $\mathrm{AFB}$ & $19(16.7)$ & $\mathrm{NA}$ & $\begin{array}{c}9 \\
(47.4)\end{array}$ & $10(52.6)$ \\
\hline $\begin{array}{l}\text { Gini and } \\
\text { Ikerionwu } \\
(1990)\end{array}$ & $\mathrm{R} / \mathrm{HA}$ & $\begin{array}{l}\text { Lower-middle } \\
\text { income }\end{array}$ & Nigeria & 4,700 & $\mathrm{HE}$ & $10(0.2)$ & $\mathrm{NA}$ & NA & NA \\
\hline $\begin{array}{l}\text { Shahzad }{ }^{62} \\
(2012)\end{array}$ & $\mathrm{R} / \mathrm{HA}$ & $\begin{array}{l}\text { Lower-middle } \\
\text { income }\end{array}$ & Pakistan & 150 & AFB, PCR, CP & $30(20)$ & NA & $\begin{array}{c}25 \\
(83.3)\end{array}$ & $5(16.7)$ \\
\hline $\begin{array}{l}\text { Shaheen et al. }{ }^{63} \\
\text { (2009) }\end{array}$ & $\mathrm{CS} / \mathrm{HA}$ & $\begin{array}{l}\text { Lower-middle } \\
\text { income }\end{array}$ & Pakistan & 534 & $\begin{array}{l}\text { CP, AFB-ZN, } \\
\text { HE }\end{array}$ & $13(2.43)$ & $13(100)$ & NA & NA \\
\hline $\operatorname{Khan}^{25}(1985)$ & $\mathrm{R} / \mathrm{HA}$ & $\begin{array}{l}\text { Upper-middle } \\
\text { income }\end{array}$ & Iran & 91 & LAP, HE & $21(23.08)$ & NA & $\begin{array}{c}15 \\
(71.4)\end{array}$ & $6(28.6)$ \\
\hline $\begin{array}{l}\text { Shallal et al } .^{64} \\
(2021)\end{array}$ & $\mathrm{PC} / \mathrm{HA}$ & $\begin{array}{l}\text { Upper-middle } \\
\text { income }\end{array}$ & Iraq & 60 & PCR, HE & $6(10)$ & $\mathrm{NA}$ & NA & NA \\
\hline $\begin{array}{l}\text { Margolis et al. }{ }^{26} \\
\text { (1992) }\end{array}$ & $\mathrm{R} / \mathrm{HA}$ & $\begin{array}{l}\text { Upper-middle } \\
\text { income }\end{array}$ & $\begin{array}{l}\text { South } \\
\text { Africa }\end{array}$ & 650 & $\mathrm{CP}$ & $40(6.15)$ & NA & $16(40)$ & $24(60)$ \\
\hline $\begin{array}{l}\text { Oosthuizen et } \\
\text { al. }^{27}(1990)\end{array}$ & $\mathrm{CS} / \mathrm{HA}$ & $\begin{array}{l}\text { Upper-middle } \\
\text { income }\end{array}$ & $\begin{array}{l}\text { South } \\
\text { Africa }\end{array}$ & 109 & $\mathrm{CP}$ & $23(21)$ & NA & NA & NA \\
\hline
\end{tabular}

$F G T B=$ female genital tuberculosis; $P I=$ primary infertility; $S I=$ secondary infertility; $C S=$ cross-sectional study; $H A=$ hospital admitted patients; $N A=N o t$ available; $P C=$ prospective cohort study; $H C=$ infertility centre admitted patient; $P C R=$ polymerase chain reaction test; $C P=$ culture proven; $H E=$ histopathological examination; $A F B=$ acid-fast bacilli test; $P=$ prospective study; $O=$ observational study; $R=$ retrospective study; $L A P=$ laparotomy; $M T$ = Mantoux test; ELISA = enzyme-linked immunosorbent assay $M H=$ menstrual history; $C C=$ case control study; $M T D=$ Mycobacterium Tuberculosis Direct test; $C B N A A T=$ cartridge based nucleic acid amplification test; $Z N=$ ZiehlNeelsen (ZN) smear microscopy. 
the eligibility criteria (total of 30,846 participants with mainly infertility complaints) were included for the systematic review and meta-analysis [Figure 1].

\section{STUDY CHARACTERISTICS}

A total of 42 studies, including 30,846 participants were included in the quantitative analysis for this meta-analysis; 2 (4.8\%) studies were from high-income countries, 4 (9.5\%) from upper-middle-income countries, 32 (76.2\%) from lower-middle-income countries and the remaining 4. (9.5\%) were from lowincome countries. Of the included studies, 17 were cross-sectional studies, 13 had a prospective study design and 12 were retrospective studies. The majority of studies were in hospital-admitted patient settings and most used diagnostic tests (only polymerase chain reaction $[\mathrm{PCR}]$ or PCR combined with other relevant test methods) [Table 1].

\section{SYNTHESIS OF RESULTS}

Out of the included study sample of 30,846 participants worldwide, there was a $20 \%$ (95\% confidence interval [CI]: 15-25\%) pooled prevalence of FGTB among infertile women. Residual heterogeneity was high ( $\mathrm{I}^{2}$ =99.94, $\left.X^{2}=2553.37 ; P<0.001\right)$. For this analysis, the random effect model was employed [Figure 2].

However, out of the 42 articles, only five, 15 and 14 articles were analysed to evaluate the pooled prevalence of overall infertility, primary and secondary infertility among FGTB patients, respectively; this resulted in a pooled prevalence rate of $88 \%$ (95\% CI: $\left.74-100 \%, I^{2}=99.91\right), 66 \%$ (95\% CI: $\left.56-76 \%, I^{2}=99.23\right)$ and 34\% (95\% CI: $\left.24-43 \%, \mathrm{I}^{2}=98.04\right)$ and $P<0.001$ each [Table 2]. In addition, the random effect model was applied because the heterogeneity was high with $P<0.001$. The publication bias was checked using the funnel plot of the forest plot and the plot was visually symmetric with Egger's test $(P=0.25)$.

Due to the very high heterogeneity level presented in FGTB among infertile women in this analysis, a two-subgroup analysis was performed to check the effect of the study's publication year and the World Bank Economical Country Classification on the pooled prevalence of FGTB among the infertile population [Table 3]. The included studies were divided as per the particular country's classification (i.e. highincome, upper-middle-income, lower-middle-income and low-income groups). The analysed data showed that the lowest income countries have a higher pooled prevalence of FGTB; conversely, the highest income countries have a lower pooled prevalence of FGTB among infertile women. The results showed pooled proportions of $5.7 \%\left(\mathrm{I}^{2}=78.56 \%\right), 14 \%\left(\mathrm{I}^{2}=86.91 \%\right)$,
$21 \%\left(\mathrm{I}^{2}=99.95 \%\right)$ and $24 \%\left(\mathrm{I}^{2}=99.48 \%\right)$ for highincome, upper-middle-income, lower-middle-income and low-income countries, respectively [Table 2].

To evaluate the effect of the study's publication year on the pooled prevalence of FGTB among infertile women, the included articles were divided into three groups. The results indicated a $10 \%, 23 \%$ and $22 \%$ pooled prevalence of FGTB among infertile women for the period before 2000, between 2001 to 2010 and between 2011 to 2021 of this study's publication year subgroups, respectively [Table 2].

\section{Discussion}

Although men significantly have the bigger burden of TB compared to women, in 2018, the WHO estimated that 3.2 million women were infected with TB and the disease is accompanied with severe consequences, especially in women of reproductive age. ${ }^{18}$ Although FGTB rarely occurs in developed countries, ${ }^{3}$ it represents an important cause of infertility in developing countries especially in countries with high TB-incidence rates. ${ }^{18}$

Recently, many published studies have investigated the prevalence of FGTB among infertile women of reproductive age which shows that the lowest prevalence was $0.45 \%$ in Nigeria and the highest prevalence was $52 \%$ in India. ${ }^{19,20}$ Worldwide, the prevalence was $24.2 \%$ in the first-published metaanalysis and systematic review in 2016. ${ }^{21}$ The current study's finding shows this prevalence to be slightly less at $20 \%$. This outcome is due to the relative progress in the availability of more sensitive TB diagnosis methods such as GeneXpert and PCR in developing countries. Moreover, there is a relative increase in the number of TB healthcare services and many countries have adopted the WHO's End TB Strategy around the globe. 22

In the current comprehensive research, the prevalence of FGTB among infertile women progressively increased over time from 10\%, 23\% and $22 \%$ in the period before 2000, between 2001 to 2010 and between 2011 to 2021, respectively. This may be due to the differences in the diagnostic methods used for FGTB which have changed over time. Surprisingly, the researchers noted that the PCR test was not used in studies published in the period before 2000 while the same diagnosis method was used by $70 \%$ and $80.8 \%$ studies for the period between 2001 to 2010 and 2011 to 2021, respectively. The utilised diagnostic methods in the analysed data were histopathological examination, ${ }^{23-25}$ culture, ${ }^{26,27}$ acid-fast bacilli test and laparotomy. ${ }^{25,28}$ According to the literature, there is 


\begin{tabular}{|c|c|c|}
\hline Author and year of publication & $\begin{array}{l}\text { Effect size } \\
(95 \% \text { CI })\end{array}$ & $\begin{array}{c}\text { Weight } \\
\text { in \% }\end{array}$ \\
\hline Ojo et al..$^{23}$ (1971) & $0.01(0.01-0.01)$ & 2.49 \\
\hline Gini and Ikerionwu ${ }^{24}(1990)$ & $0.00(0.00-0.00)$ & 2.49 \\
\hline Emembolu $^{28}(1989)$ & $0.17(0.10-0.24)$ & 2.37 \\
\hline $\operatorname{Khan}^{25}(1985)$ & $0.23(0.14-0.32)$ & 2.31 \\
\hline Margolis et al. ${ }^{26}$ (1992) & $0.05(0.04-0.08)$ & 2.48 \\
\hline Oosthuizen et al. ${ }^{27}$ (1990) & $0.21(0.13-0.29)$ & 2.36 \\
\hline Chattopadhyay et al. ${ }^{38}$ (1986) & $0.04(0.03-0.05)$ & 2.48 \\
\hline Ali and Abdallah ${ }^{40}(2012)$ & $0.01(0.01-0.01)$ & 2.49 \\
\hline Abebe et al. ${ }^{13}$ (2004) & $0.54(0.45-0.83)$ & 1.82 \\
\hline Jindal $^{10}(2006)$ & $0.07(0.05-0.08)$ & 2.49 \\
\hline Shaheen et al. ${ }^{63}$ (2009) & $0.02(0.01-0.04)$ & 2.48 \\
\hline Ojo et al. ${ }^{19}$ (2008) & $0.00(-0.00-0.01)$ & 2.49 \\
\hline Gupta et al. ${ }^{60}(2007)$ & $0.27(0.20-0.34)$ & 2.37 \\
\hline Singh et al. ${ }^{43}$ (2008) & $0.48(0.40-0.57)$ & 2.32 \\
\hline Nezar et al. ${ }^{42}$ (2009) & $0.05(0.03-0.08)$ & 2.48 \\
\hline Kumar et al. ${ }^{6}(2008)$ & $0.39(0.33-0.45)$ & 2.41 \\
\hline Chowdhury et al. ${ }^{47}$ (2010) & $0.45(0.40-0.49)$ & 2.44 \\
\hline Saraswat et al. ${ }^{48}$ (2010) & $0.21(0.14-0.28)$ & 2.36 \\
\hline Abdissa et al. ${ }^{4}$ (2018) & $0.05(0.02-0.09)$ & 2.46 \\
\hline Abdelrub and $\mathrm{Al} \mathrm{Harazi}{ }^{41}(2015)$ & $0.31(0.24-0.38)$ & 2.36 \\
\hline Mohakul et al. ${ }^{5}$ (2015) & $0.39(0.30-0.48)$ & 2.28 \\
\hline Shahzad $^{62}$ (2012) & $0.20(0.14-0.25)$ & 2.39 \\
\hline Sankar et al. ${ }^{44}$ (2013) & $0.25(0.22-0.29)$ & 2.46 \\
\hline Goel et al.53 (2013) & $0.04(0.02-0.05)$ & 2.48 \\
\hline Malhotra et al. ${ }^{49}$ (2012) & $0.25(0.22-0.29)$ & 2.46 \\
\hline Patil et al.52 (2015) & $0.01(-0.01-0.02)$ & 2.48 \\
\hline Shrivastava and Patel $^{7}$ (2014) & $0.39(0.33-0.45)$ & 2.38 \\
\hline Parikh KP et al. ${ }^{59}(2014)$ & $0.12(0.03-0.21)$ & 2.30 \\
\hline Bhanothu et al. ${ }^{51}$ (2014) & $0.28(0.23-0.34)$ & 2.42 \\
\hline Mahajan et al. ${ }^{45}$ (2016) & $0.41(0.34-0.48)$ & 2.36 \\
\hline Sethi et al. ${ }^{46}(2016)$ & $0.23(0.18-0.27)$ & 2.43 \\
\hline GajBhIye et al..$^{50}$ (2019) & $0.12(0.03-0.21)$ & 2.30 \\
\hline Kamal et al. ${ }^{54}(2020)$ & $0.27(0.18-0.36)$ & 2.31 \\
\hline Shende et al. ${ }^{57}$ (2017) & $0.27(0.19-0.35)$ & 2.34 \\
\hline Ohri et al. ${ }^{58}(2016)$ & $0.18(0.07-0.29)$ & 2.23 \\
\hline Chatterjee et al..$^{65}$ (2018) & $0.02(-0.01-0.04)$ & 2.48 \\
\hline Gurjar et al. ${ }^{20}(2018)$ & $0.52(0.42-0.52)$ & 2.26 \\
\hline Kanti et al..$^{55}$ (2021) & $0.03(-0.01-0.08)$ & 2.43 \\
\hline Meenu et al. ${ }^{56}(2020)$ & $0.42(0.34-0.50)$ & 2.33 \\
\hline Rajaramet al. ${ }^{61}$ (2016) & $0.28(0.15-0.40)$ & 2.14 \\
\hline Shallal et al. ${ }^{64}$ (2021) & $0.10(0.02-0.18)$ & 2.36 \\
\hline Tal et al..$^{39}$ (2020) & $0.08(0.05-0.11)$ & 2.47 \\
\hline Overall & $0.20(0.15-0.25)$ & \\
\hline
\end{tabular}

Heterogeneity: $\mathrm{T}^{2}=0.02, \mathrm{I}^{2}=99.94 \%, \mathrm{H}^{2}=1745.13$

Test of $\theta 1=\theta 1: \mathrm{Q}(41)=2553.37 ; P=0.00$

Test of $\theta=\theta: \mathrm{z}=7.94 ; P=0.00$

$$
\begin{array}{lllll}
\hline 0 & .2 & .3 & .4 & .5
\end{array}
$$

Figure 2: Forest plot (random-effects model) showing the pooled prevalence of female genital tuberculosis among infertile women. ${ }^{4-7,10,13,19,20,23-28,38-65}$

$C I=$ confidence interval. 
Table 2: Pooled prevalence of infertility among female genital tuberculosis (FGTB) patients, pooled proportion of FGTB among infertile women based on the World Bank country economic classification and subgroup analysis of FGTB among infertile women based on year of publication*

\begin{tabular}{|c|c|c|c|c|c|c|c|}
\hline \multirow[t]{2}{*}{ Subgroup classification } & \multirow[t]{2}{*}{ Subgroup } & \multirow{2}{*}{$\begin{array}{l}\text { Number } \\
\text { of studies }\end{array}$} & \multirow{2}{*}{$\begin{array}{c}\text { Total } \\
\text { number } \\
\text { of } \\
\text { patients }\end{array}$} & \multirow{2}{*}{$\begin{array}{c}\text { FGTB } \\
\text { proportion } \\
\text { in } \% \\
(95 \% \mathrm{CI})\end{array}$} & \multirow{2}{*}{$\begin{array}{c}\text { Infertility } \\
\text { proportion } \\
\text { in \% } \\
(95 \% \mathrm{CI})\end{array}$} & \multicolumn{2}{|c|}{ Heterogeneity } \\
\hline & & & & & & $I^{2}$ in \% & $P$ value \\
\hline \multirow[t]{3}{*}{$\begin{array}{l}\text { Type of infertility (among FGTB } \\
\text { patients) }\end{array}$} & $\begin{array}{l}\text { Pooled } \\
\text { infertility }\end{array}$ & 5 & 430 & - & $88(74-100)$ & 99.912 & $>0.001$ \\
\hline & $\begin{array}{l}\text { Primary } \\
\text { infertility }\end{array}$ & 15 & 560 & - & $66(56-76)$ & 99.226 & $>0.001$ \\
\hline & $\begin{array}{l}\text { Secondary } \\
\text { infertility }\end{array}$ & 14 & 558 & - & $34(24-43)$ & 98.039 & $>0.001$ \\
\hline \multirow{4}{*}{$\begin{array}{l}\text { World Bank country economic } \\
\text { classification (among infertile } \\
\text { patients) }\end{array}$} & $\begin{array}{l}\text { High } \\
\text { income }\end{array}$ & 2 & 1268 & $5.7(2.3-9.1)$ & - & 78.56 & $>.001$ \\
\hline & $\begin{array}{l}\text { Upper- } \\
\text { middle } \\
\text { income }\end{array}$ & 4 & 910 & $14(6-23)$ & - & 86.91 & $>0.001$ \\
\hline & $\begin{array}{l}\text { Lower- } \\
\text { middle } \\
\text { income }\end{array}$ & 32 & 25,562 & $21(15-27)$ & - & 99.95 & $>0.001$ \\
\hline & $\begin{array}{l}\text { Low } \\
\text { income }\end{array}$ & 4 & 3,106 & $24(3-52)$ & - & 99.48 & 0.084 \\
\hline \multirow{3}{*}{$\begin{array}{l}\text { Year of publication } \\
\text { (among infertile patients) }\end{array}$} & $<2000$ & 7 & 18,530 & $10(3-17)$ & - & 99.96 & $<0.001$ \\
\hline & $2001-2010$ & 11 & 7,718 & $23(10-36)$ & - & 99.93 & $<0.001$ \\
\hline & $2011-2021$ & 24 & 4,623 & $22(16-27)$ & & 97.98 & $<0.001$ \\
\hline
\end{tabular}

$F G T B=$ female genital tuberculosis; $C I=$ confidence interval.

*Out of 42 studies 34 were analysed for type of infertility (subgroup among FGTB patients) and the remaining studies were excluded as there was no mention of the prevalence of infertility, primary infertility or secondary infertility.

no gold-standard test for FGTB as this depends on the facilities' test protocol. However, different FGTB testing methods have been providing various results of the disease rate among infertile women. ${ }^{20}$ The increase of the prevalence of FGTB among infertile women is due to the previously mentioned reasons, including utilisation of modern TB diagnosis methods and adopting the WHO's End TB Strategy. ${ }^{18}$ Furthermore, the global funds on TB control have substantially increased in recent decades. ${ }^{29}$

The present study reveals that the prevalence of FGTB is inversely proportional to the economic situation of the country. The lowest prevalence was $5.7 \%$ in the high-income countries while the highest prevalence was $24 \%$ in the low-income countries. The upper-middle-income and lower-middle-income countries showed $14 \%$ and $22 \%$, respectively. Although, there was no published data to describe the rate of FGTB among infertile women in different countries based on their economic status, other studies have shown that FGTB is associated with PTB and EPTB as secondary infections. ${ }^{2,3}$ This outcome may be due to the delay of TB diagnosis and other sociocultural reasons. In line with this, Getnet et al. reported that $42 \%$ of PTB diagnoses were delayed for a varied period of time (a month to a year) in low-income and middleincome countries' setting. ${ }^{30}$ Furthermore, MacPherson et al. indicated that 4-38\% of TB-patients were lost to follow-up for treatment in the same setting. ${ }^{31}$ In the Middle East and North Africa, factors such as being female and having a low income per capita is relatively reflected in the delay of TB diagnosis; ${ }^{32}$ although, the proportions are $1.24 \%$ and $1.26 \%$, respectively, it has considerable impact on FGTB incidence. In addition, the high incidence of FGTB in low- and middleincome settings is due to factors such as the higher rate of losses to follow-up with TB or EPTB treatment and the relatively negative experiences of TB patients and their level of satisfaction with their healthcare system. ${ }^{33,34}$ Moreover, poverty and the high cost of the accurate diagnosis of FGTB in developing countries have a largely negative effect on FGTB control and treatment. ${ }^{35,36}$ In accordance with this, Cazabon et al. reported that $32 \%$ and $46 \%$ of TB patients had a negative experience and were dissatisfied with healthcare providers and TB services, respectively. ${ }^{34}$

The findings of the current study reveal that the pooled prevalence of infertility among overall FGTBpatients was very high (88\%). Of this, the pooled prevalence of primary infertility was higher than 
that of secondary infertility among FGTB patients. Although these results are in agreement with other meta-analysis findings done by Chaman-Ara et al. (who reported $70.7 \%, 75.7 \%$ and $24.3 \%$ for infertility among FGTB patients, primary infertility and secondary infertility, respectively), the present study showed a slight increase in the pooled prevalence infertility and secondary infertility incidence among FGTB patients. On the other hand, the rate of primary infertility decreased over time. ${ }^{37}$

To achieve the WHO End TB Strategy goal to eliminate catastrophic costs for TB-affected households by 2030 as Sustainable Development Goal target, a more thorough clinical investigation should be administrated at the level of TB and infertility clinics, particularly in low- and lower-income settings. ${ }^{18}$

This review is subject to certain limitations. Articles published in languages other than English were excluded and the study population included only infertile women of reproductive age. Some grey literature may have also been missed. During the data collection process, no published studies investigating the incidence of FGTB among infertile women from the continents of Australia, Europe or South America were found. The likelihood for publication bias is high.

\section{Conclusion}

The results of this meta-analysis found that the pooled prevalence of FGTB among infertile women is $20 \%$, and the pooled prevalence of overall infertility, primary infertility and secondary infertility among FGTB patients globally is $88 \%, 66 \%$ and $34 \%$, respectively. In the last two decades, the FGTB incidence rate was increasing gradually. The biggest burden of FGTB is reported in the low- and lower-middle-income countries with a pooled prevalence of $46 \%$ globally.

\section{ACKNOWLEDGEMENTS}

The authors would like to thank the Pan African University, Institute Life and Earth Sciences (Including Health and Agriculture), the African Union for financial support and University of Ibadan for hosting this Ph.D. programme.

\section{AUTHORS' CONTRIBUTIONS}

MA, AM, AI, CA, AB and SH conceived and designed the review. MA, AM and $\mathrm{SH}$ carried out the draft of the manuscript and MA is the guarantor of the review. MA, AM, AI, CA, AB and $\mathrm{SH}$ developed the search strings. MA, AM and SH screened, evaluated and selected the studies as well as extracted the data. $\mathrm{AI}, \mathrm{CA}$ and $\mathrm{AB}$ evaluated the quality of the studies.
MA and AM carried out the statistical analysis and interpretation. MA, AM, AI, CA, AB and $\mathrm{SH}$ rigorously reviewed the manuscript. All authors approved the final version of the manuscript.

\section{References}

1. World Health Organization. Global tuberculosis report 2020. From: https://www.who.int/publications/i/item/9789240013131

2. Grace GA, Devaleenal DB, Natrajan M. Genital tuberculosis in females. Indian J Med Res 2017; 145:425-36. https://doi. org/10.4103/ijmr.IJMR_1550_15.

3. Parvez R, Sugunan AP, Vijayachari P, Burma SP, Mandal A, Saha MK, et al. Prevalence of female genital tuberculosis, its risk factors and associated clinical features among the women of Andaman Islands, India: A community-based study. Public Health 2017; 148:56-62. https://doi.org/10.1016/j.puhe.2017.03.001.

4. Abdissa S, Abebe T, Ameni G, Teklu S, Bekuretsion Y, Abebe M, et al. Endometrial tuberculosis among patients undergoing endometrial biopsy at Tikur Anbesa specialized hospital, Addis Ababa, Ethiopia. BMC Infect Dis 2018; 18:1-8. https://doi. org/10.1186/s12879-018-3202-x.

5. Mohakul SK, Beela VRK, Tiru P. Hysteroscopy findings and its correlation with latent endometrial tuberculosis in infertility. Gynecological Surgery 2015; 12:31-9. https://doi.org/10.1007/ s10397-014-0865-1.

6. Kumar P, Shah NP, Singhal A, Chauhan DS, Katoch VM, Mittal S, et al. Association of tuberculous endometritis with infertility and other gynecological complaints of women in India. J Clin Microbiol 2008; 46:4068-70. https://doi.org/10.1128/JCM.01162-08.

7. Shrivastava G, Patel K. Genital tuberculosis: Evaluating microscopy, culture, histopathology and PCR for diagnosis all play their role. Int J Curr Microbiol App Sci 2014; 3:439-45.

8. Fatima T, Hasan R, Malik FR, Ahmed I, Bartlett LA, Gravett MG, et al. Female genital tuberculosis in Pakistan - A retrospective review of 10-year laboratory data and analysis of 32 cases. Int J Mycobacteriol 2021; 10:66-70. https://doi.org/10.4103/ijmy. ijmy_6_21.

9. A AA, Ahmed M, Oladokun A. Prevalence of infertility in Sudan: A systematic review and meta-analysis. Qatar Med J 2021; 2021:47. https://doi.org/10.5339/qmj.2021.47.

10. Jindal UN. An algorithmic approach to female genital tuberculosis causing infertility. The Int J Tuberc Lung Dis 2006; 10:1045-50.

11. Namavar Jahromi B, Parsanezhad ME, Ghane-Shirazi R. Female genital tuberculosis and infertility. Int J Gynaecol Obstet 2001; 75:269-72. https://doi.org/10.1016/s0020-7292(01)00494-5.

12. Bhanothu V, Theophilus JP, Reddy PK, Rozati R. Occurrence of female genital tuberculosis among infertile women: a study from a tertiary maternal health care research centre in South India. Europ Eur J Clin Microbiol Infect Dis 2014; 33:1937-49. https://doi.org/10.1007/s10096-014-2164-1.

13. Abebe M, Lakew M, Kidane D, Lakew Z, Kiros K, Harboe, et al. Female genital tuberculosis in Ethiopia. Int J Gynaecol Obstet 2004; 84:241-6. https://doi.org/10.1016/j.ijgo.2003.11.002.

14. Dai W, Ma L, Cao Y, Wu D, Yu T, Zhai J. In vitro fertilization outcome in women with endometrial tuberculosis and tubal tuberculosis. Gynecol Endocrinol 2020; 36:819-823. https:// doi.org/10.1080/09513590.2019.1702639.

15. Liberati A, Altman DG, Tetzlaff J, Mulrow C, Gøtzsche PC, Ioannidis JP, et al. The PRISMA statement for reporting systematic reviews and meta-analyses of studies that evaluate health care interventions: Explanation and elaboration. J Clin Epidemiol 2009; 62:e1-34. https://doi.org/10.1016/j.jclinepi.2009.06.006. 
16. Porritt K, Gomersall J, Lockwood. Study selection and critical appraisal: the steps following the literature search in a systematic review. CJAJN 2014; 114:47-52. https://doi.org/10.1097/01. NAJ.0000450430.97383.64.

17. Higgins JPT, Thompson SG, Deeks JJ, Altman DG. Measuring inconsistency in knowledgebases. BMJ 2003; 327:557-60. https://doi.org/10.1136/bmj.327.7414.557.

18. World Health Organization. Global tuberculosis report 2018 From: https://www.who.int/publications/i/item/9789241565646

19. Ojo BA, Akanbi AA, Odimayo MS, Jimoh AK. Endometrial tuberculosis in the Nigerian middle belt: An eight-year review. Trop Doc 2008; 38:3-4. https://doi.org/10.1258/td.2007.052090.

20. Gurjar K, Meena KL, Rajoria L, Sharma N. Comparison of diagnostic efficacy of USG, Tuberculin test, Nucleic acid amplification test (PCR) \& histopathology for diagnosis of genital tuberculosis in infertile women, assuming culture as gold standard. IMJH 2018; 4:138-43.

21. Chaman-Ara K, Bahrami MA, Bahrami E, BahramiS, BahramiMN, Moosazadeh M, et al. Prevalence of genital tuberculosis among infertile women: A systematic review and meta-analysis. Int J Med Res Health Sci 2016; 5:208-15.

22. World Health Organization. Framework for implementing the "End TB Strategy" in the African Region 2016 - 2020. From: https://www.afro.who.int/publications/framework-implem enting-end-tb-strategy-african-region-2016-2020

23. Ojo OA, Onifade A, Akande EO, Bannerman RH. The pattern of female genital tuberculosis in Ibadan. Isr J Med Sci 1971; 7:280-7.

24. Gini PC, Ikerionwu SE. Incidental tuberculous endometritis in premenstrual curettings from infertile women in Eastern Nigeria. Int J Gynaecol Obstet $1990 ; 31: 141-4$. https://doi. org/10.1016/0020-7292(90)90711-s.

25. Khan SM. Incidence of genital tuberculosis in infertile women. J Pak Med Assoc 1985; 35:280-1.

26. Margolis K, Wranz PA, Kruger TF, Joubert JJ, Odendaal HJ. Genital tuberculosis at Tygerberg Hospital--prevalence, clinical presentation and diagnosis. S Afr Med J 1992; 81:12-15.

27. Oosthuizen AP, Wessels PH, Hefer JN. Tuberculosis of the female genital tract in patients attending an infertility clinic. S Afr Med J 1990; 77:562-4.

28. Emembolu JO. Endometrial flora of infertile women in Zaria, Northern Nigeria. Int J Gynecol Obstet 1989; 30:155-9. https:// doi.org/10.1016/0020-7292(89)90310-x.

29. Floyd K, Fitzpatrick C, Pantoja A, Raviglione M. Domestic and donor financing for tuberculosis care and control in lowincome and middle-income countries: an analysis of trends, 2002-11, and requirements to meet 2015 targets. Lancet Glob Health 2013; 1:e105-15. https://doi.org/10.1016/S2214109X(13)70032-9.

30. Getnet F, Demissie M, Assefa N, Mengistie B, Worku A. Delay in diagnosis of pulmonary tuberculosis in low-and middleincome settings: Systematic review and meta-analysis. BMC Pulm Med 2017; 17:1-15. https://doi.org/10.1186/s12890-0170551-y.

31. MacPherson P, Houben RMGJ, Glynn JR, Corbett EL, Kranzer K. Pre-treatment loss to follow-up in tuberculosis patients in lowand lower-middle-income countries and high-burden countries A systematic review and meta-analysis. Bull World Health Organ 2013; 92:126-38. https://doi.org/10.2471/BLT.13.124800.

32. Eltayeb D, Pietersen E, Engel M, Abdullahi L. Factors associated with tuberculosis diagnosis and treatment delays in Middle East and North Africa: A systematic review. East Mediterr Health J 2020; 26:477-86. https://doi.org/10.26719/2020.26.4.477.

33. Ade S, Harries AD, Trébucq A, Ade G, Agodokpessi G, Adjonou $\mathrm{C}$, et al. National profile and treatment outcomes of patients with extrapulmonary tuberculosis in Bénin. PLoS One 2014; 9:e95603. https://doi.org/10.1371/journal.pone.0095603.
34. Cazabon D, Pande T, Sen P, Daftary A, Arsenault C, Bhatnagar H, et al. User experience and patient satisfaction with tuberculosis care in low-and middle-income countries: a systematic review. J Clin Tuberc Other Mycobact Dis 2020; 19:100154. https://doi. org/10.1016/j.jctube.2020.100154.

35. Lu C, Liu Q, Sarma A, Fitzpatrick C, Falzon D, Mitnick CD. A systematic review of reported cost for smear and culture tests during multidrug-resistant tuberculosis treatment. PLoS One 2013; 8:e56074. https://doi.org/10.1371/journal.pone.0056074.

36. Lund C, De Silva M, Plagerson S, Cooper S, Chisholm D, Das J, et al. Poverty and mental disorders: Breaking the cycle in lowincome and middle-income countries. Lancet 2011; 378:1502-14. https://doi.org/10.1016/S0140-6736(11)60754-X

37. Chaman-Ara K, Bahrami MA, Moosazadeh M, Bahrami E. Prevalence of infertility in women with genital tuberculosis: a systematic review and meta-analysis. IJBS 2017; 11:21-7. https://doi.org/10.15562/ijbs.v11i1.133.

38. Chattopadhyay SK, Sengupta BS, Edrees YB, Al-Meshari AA. The pattern of female genital tuberculosis in Riyadh, Saudi Arabia. Br J Obstet Gynaecol 1986; 93:367-71.

39. Tal R, Lawal T, Granger E, Simoni M, Hui P, Buza N, et al. Genital tuberculosis screening at an academic fertility center in the United States. Am J Obstet Gynecol 2020; 223:737.e1-10. https://doi.org/10.1016/j.ajog.2020.05.045

40. Ali AA, Abdallah TM. Clinical presentation and epidemiology of female genital tuberculosis in eastern Sudan. Int J Gynaecol Obstet 2012; 118:236-8. https://doi.org/10.1016/j. ijgo.2012.04.005

41. Abdelrub AS, Al Harazi AH. Genital tuberculosis is common among females with tubal factor infertility: Observational study. Alexandria J Med 2015; 51:321-4. https://doi.org/10.1016/j. ajme.2014.11.004.

42. Nezar M, Goda H, El-Negery M, El-Saied M, Wahab AA, Badawy AM. Genital tract tuberculosis among infertile women: an old problem revisited. Arch Gynecol Obstet 2009; 280:787-91. https://doi.org/10.1007/s00404-009-1000-9.

43. Singh N, Sumana G, Mittal S. Genital tuberculosis: A leading cause for infertility in women seeking assisted conception in North India. Arch Gynecol Obstet 2008; 278:325-7. https:// doi.org/10.1007/s00404-008-0590-y.

44. Sankar MM, Kumar P, Munawwar A, Kumar M, Singh J, Singh A, et al. Usefulness of multiplex PCR in the diagnosis of genital tuberculosis in females with infertility. Eur J Clin Microbiol Infect Dis 2013; 32:399-405. https://doi.org/10.1007/s10096012-1755-y.

45. Mahajan N, Naidu P, Kaur SD. Insight into the diagnosis and management of subclinical genital tuberculosis in women with infertility. J Hum Reprod Sci 2016; 9:135-44. https://doi. org/10.4103/0974-1208.192043

46. Sethi S, Dhaliwal L, Dey P, Kaur H, Yadav R. Loop-mediated isothermal amplification assay for detection of Mycobacterium tuberculosis complex in infertile women. Indian J Med Microbiol 2016; 34:322-7. https://doi.org/10.4103/0255-0857.188323.

47. Chowdhury RG, Paine SK, Bhattacharjee B, Chatterjee S. Infestation of endometrium by mycobacterium tuberculosis bacilli-cause of reproductive failure. Al Ameen J Med Sci 2010; 3:322-31

48. Saraswat P, Swarankar ML, Bhandari A, Soni RR. Detection of active female genital tuberculosis by molecular method. Int J Pharma Bio Sci 2010; 1:328-34.

49. Malhotra B, Sinha P, Hooja S, Vyas L. Rapid diagnosis of genital tuberculosis by real time polymerase chain reaction. J South Asian Feder Obst Gynae 2012; 4:39-42. https://doi. org/10.5005/jp-journals-10006-1170.

50. GajBhIye SB, Bhakre JB, Damle AS. Assessment of Endometrial Specimens for Tubercular Infection among Infertile Women using DNA-PCR. J Clin Diagnostic Res 2019; 13:DC01-5. https://doi.org/10.7860/JCDR/2019/42587.13208. 
51. Bhanothu V, Theophilus JP, Rozati R. Use of endo-ovarian tissue biopsy and pelvic aspirated fluid for the diagnosis of female genital tuberculosis by conventional versus molecular methods. PLoS One 2014; 9:e98005. https://doi.org/10.1371/ journal.pone.0098005.

52. Patil AD, Shinde SK, Sachdeva GM, Pasi AR, Chitlange SM, Chandhiok $\mathrm{N}$, et al. Is testing of menstrual blood justifiable for diagfnosis of endometrial tuberoculosis among infertile women. Ind J Obstet Gynecol Res 2015; 2:103-7.

53. Goel G, Khatuja R, Radhakrishnan G, Agarwal R, Agarwal S, Kaur I. Role of newer methods of diagnosing genital tuberculosis in infertile women. Indian J Pathol Microbiol 2013; 56:155-7. https://doi.org/10.4103/0377-4929.118670.

54. Kamal S, Singh V, Singh S. A clinical study of association of genital tuberculosis with infertility in a tertiary centre of Jharkhand, India. Int J Reproduction, Contraception, Obstet Gyne 2020 9:120-6. https://doi.org/10.18203/2320-1770.ijrcog20196008.

55. Kanti V, Seth S, Gupta S, Verma V, Singh A, Maurya G, et al. Comparison of the Efficacy of the Cartridge-Based Nucleic Amplification (CBNAAT)/Xpert Test and Histology of Genital Tissues in Diagnosing Female Genital Tuberculosis. Cureus 2021; 13:e15000. https://doi.org/10.7759/cureus.15000.

56. Meenu S, Ramalingam S, Sairam T, Appinabhavi A, Panicker S, Oommen S, et al. Comparison of Polymerase Chain Reaction (PCR), Microbiological and Histopathological Observations in the Diagnosis of Endometrial Tuberculosis. J Obstet Gynaecol India 2020; 70:510-15. https://doi.org/10.1007/s13224-020-01367-9.

57. Shende P, Valecha SM, Gandhewar M, Dhingra D. Genital tuberculosis and infertility. Int J Reprod Contracept Obstet Gynecol 2017; 6:3514-18.
58. Ohri S, Patil SK, Patil A, Patil Y, Kshirsagar NS. Study of genital tuberculosis in infertile women. Age 2016; 21:F1-3.

59. Parikh KP, Deshpande HG, Madkar CS, Jethani S. Study to find out the incidence of Genital Tuberculosis as a cause for female infertility in Semi-Urban population. J Evol Med Dent Sci 2014; 3:3223-7. https://doi.org/10.14260/jemds/2014/2271.

60. Gupta N, Sharma JB, Mittal S, Singh N, Misra R, Kukreja M. Genital tuberculosis in Indian infertility patients. Int J Gynaecol Obstet 2007; 97:135-8. https://doi.org/10.1016/j. ijgo.2006.12.018

61. Rajaram S, Gupta P, Gupta B, Kaur IR, Goel N. Laparoscopy in the diagnosis of tuberculosis in chronic pelvic pain. Int J Mycobacteriol 2016; 5:318-23. https://doi.org/10.1016/j. ijmyco.2016.06.016

62. Shahzad S. Investigation of the prevalence of female genital tract tuberculosis and its relation to female infertility: An observational analytical study. Iran J Reprod Med 2012; 10:581-8.

63. Shaheen R, Subhan F, Tahir F. Epidemiology of genital tuberculosis in infertile population. J Pak Med Assoc 2006; 56:306-9.

64. Shallal MM, Al-Asadi FAS, Mizaal MI. Detection of Endometrial TB in Patients with AUB using PCR Method of Assessment of Menstrual Blood Flow of Iraqi Females. Indian J Forensic Med Toxicol 2021; 15:1225. https://doi.org/10.37506/ ijfmt.v15i1.13584.

65. Chatterjee T., Basak AK. Mycobacterium tuberculosis and non-tubercular mycobacterium infection in women with unexplained infertility from eastern India. Int J Reprod Biomed 2018; 16:557-62 